\title{
MINIMALLY INVASIVE GASTROJEJUNAL BYPASS IS THE “GOLDEN STANDARD” IN BARIATRIC SURGERY OF MORBID OBESITY
}

\section{Kolesnykov Ye. B.}

\section{INTRODUCTION}

Morbid obesity became major health problem in the US and growing worldwide becoming a pandemic. More than 10 million people are currently morbidly obese in the USA ${ }^{1}$. Severe obesity places a large financial burden on the health care system due to co-morbid conditions such as diabetes mellitus, obstructive sleep apnea, hypertension, hyperlipidemia, degenerative joint disease and certain cancers. Dietary measures have poor results with only a $1 \%$ success rate for long term weight maintenance. Bariatric surgery offers the only effective treatment for life.

The National Institute of Health consensus conference on obesity in 1991 recommended surgical treatment as an option for patients with BMI over 40 and for those with BMI over 35 with obesity related co-morbidities. Recent new surgical techniques improved perioperative care made bariatric surgery safe, widely accepted and popular. In 2020 more than 250000 bariatric operations were performed in the US. With worldwide increase of these operations, the medical community and health insurance companies are becoming more familiar and supportive of bariatric surgery.

Currently, $64 \%$ of the US population and $42 \%$ in Ukraine are overweight or obese. Obesity is now recognized as a disease that has spread throughout the world in the form of a pandemic ${ }^{2}$. This disease in most cases for life, there are no reliable ways of drug treatment for it, often there are medical, cosmetic and psychological problems (depression, turmoil in family life, low self-esteem, infertility, etc.).This disease shortens life due to the accompanying pathological conditions (type 2 diabetes, hypertension, cardiovascular complications, thromboembolism, etc.), and also increases

\footnotetext{
${ }^{1}$ Halmi D, Anez O, Kolesnikov E. Mini-open Roux-en-Y gastric bypass is a safe and cost-effective alternative to the open and laparoscopic bariatric surgery. XXXIV World Congress of the International College of Surgeons, October 2004, Quito, Ecuador. International Proceedings MEDIMOND, Italy, 2004, p. 105.

2 Halmi D, Anez O, Kolesnikov E. Mini-open Roux-en-Y gastric bypass: Complications and Difficulties. XXXIV World Congress of the International College of Surgeons, October 2004, Quito, Ecuador. International Proceedings, MEDIMOND, Italy,2004, p. 109.
} 
the risk of malignant neoplasms ${ }^{3}$. Therapy of morbid obesity with medications, as a rule, is ineffective - success is achieved only in $1 \%$ of cases. Surgical treatment of morbid obesity is optimal. When using the methods of bariatric surgery, the success in long-term control of the patient's weight reaches $70-80 \%$. Surgical treatment allows to achieve resolution of the majority of concomitant pathological obesity syndromes. In this regard, there is currently an increase in the number of bariatric surgeries both throughout the world and in our country.

Surgical procedures on morbidly obese patients are technically challenging, and the pre- and postoperative care requires expertise from the surgical team as well as from the other medical consultants. There is a steep learning curve for the procedures during which significant and serious complications can occur. This learning is about 100 operations for the laparoscopic Roux-en-Y Gastric Bypass (RYGB). Thus, less complex procedures became popular such as the laparoscopic adjustable gastric banding due to the lower risk of serious complications and because it is technically easier to perform. However, the weight loss and long term maintenance seems to be inferior to the RYGB. The expected weight loss after RYGB is about $65-75 \%$ of the excess body weight with successful long term weight maintenance in approximatelly $80 \%$ of all operated patients.

\section{Materials and research methods in morbidly obese patients}

In our bariatric surgery center we (D. Halmi, O. Anez, E. Kolesnikov) created in 2004 a mini-invasive bariatric operation, which was named Mini-open Roux-en-Y Gastric Bypass for the surgical treatment of morbidly obese patients.

Operation is, in fact, a gastrojejunal bypass using stapling technique, performed through the mini-laparotomy incision. This operation was shown at numerous world, American and European congresses and was recognized by the International Federation of Bariatric Surgeons as the "gold standard" in the treatment of morbid obesity ${ }^{4}$.

To date, the participants in the development of this method have operated (in the USA and Ukraine) 6,000 patients ${ }^{5}$. The demographic composition of patients: men $-18.3 \%$, women $-81.7 \%$; average age: $42 \pm 6$ years (16-68); average weight: $138 \pm 12.7$ (97-273) $\mathrm{kg}$; mean body mass index (BMI):

\footnotetext{
${ }^{3}$ Interdisciplinary European Guidelines on Metabolic and Bariatric Surgery. Fried M, Yumuk V, Oppert J-M, Scopinaro N et al. The European Journal of obesity, Obes Facts 2013;6:449

${ }^{4}$ Halmi D, Anez O, Kolesnikov E. Mini-open Roux-en-Y gastric bypass: 1200 anastomoses without leak. Obesity Surgery, 14, 8, 2004, p. 900.

${ }^{5}$ Halmi D, Anez O, Kolesnikov E. Mini-open Roux-en-Y gastric bypass as the operation of choice for the majority of morbidly obese patients. Obesity surgery, 15, 2005, p. 960.
} 
$46.6 \pm 8.9 \mathrm{~kg} / \mathrm{m} 2$ (35-86). BMI: $35-39-8 \%, 40-49-61.2 \%, 50-59-24.1 \%$, $\geq 60-5.7 \%, \geq 70-0.8 \%, \geq 80 \%$

\section{Minimaly invasive surgical technique}

Surgical treatment of morbid obesity was performed using a mini-open or laparoscopic method, however, the main stages of gastro-jejunal shunting remain the same, regardless of the method of operation. The main stages of the proposed by us method of gastrointestinal bypass, which includes a restrictive component and moderate malabsorption, are: 1) the formation of a small ventricle, with a volume of $25-30 \mathrm{ml}$ using a linear stapler; 2) proximal $(90-135 \mathrm{~cm})$ gastrointestinal bypass grafting Roux-en $Y$; 3) Side-to-side stapler jejuno-jejunal anastomosis; 4) Imposition of gastrojejunostomy with a circular stapler (ILS-21 or $25 \mathrm{~mm}$ ) ${ }^{6}$.

To ensure the protection of the wound edges from contact with gastrointestinal contents and wound retraction, we use the "Protractor ring", which is two rubber rings (flat and round) connected by a polypropylene membrane $^{7}$ (Figure 1).

An important surgical maneuver is the formation of a tunnel with the index finger around the esopageal-gastric junction to pass the rubber tube, which is used as a guide for the TX 60 (Ethicon), USA linear stapler (Figure 2 ). The creation of a small stomach with a linear stapler can be done in two ways. The first option - stapling with three stapler (TX60) lines without cutting the stomach and the second option - with transection of the stomach using Endo GIA Flex-45 or Echelon stapler. When performing the first option, a TX60 linear stapler (Ethicon, USA) is used, with which the stomach is stapled three times from the lesser curvature $6 \mathrm{~cm}$ from the gastroesophageal junction towards the angle of His.

Use 2 rows of green staples $(4.8 \mathrm{~mm}$ ) and one blue. ( Figure 3) At the lower end of the stapler line, a holding suture (Prolene 2.0) is applied. In the lower part of the formed small pouch of the stomach, with a volume of 30-50 ml, gastrotomy is performed with a vertical incision $(1.5-2.0 \mathrm{~cm})$. An envil from a circular stapler (ILS 21 or ILS25) is installed in the upper part of the gastrotomy opening and is tightly sutured with 5-6 linear (nonpurse-string) silk sutures (3.0). The first stage of the operation ends here.

${ }^{6}$ Halmi D, Anez O, Kolesnikov E. Roux-en-y gastric bypass: gastro-jejunostomy technique and complications. $2^{\text {nd }}$ European IFSO Congress of Bariatric and Plastic Surgery, April 27-29, 2006, Lyon, France Obesity Surgery, 16, (4) 2006, 404.

Halmi D, Kolesnikov E. Roux-en-y gastric bypass with concomitant cholecystectomy through the mini-laparotomy incision, 7-th World Congress of the International Hepato-Pancreato Billiary Association in Edinburgh, Scotland, September 3-7, 2006, HPB journal, Vol. 8; No 2 supl, Sept 2006, p. 101.

${ }^{8}$ Halmi D, Kolesnikov E. Pre-operative placement of retrievable inferior vena cava filters in bariatric surgery. Surg for Obes and Rel Dis, Vol. 3, No. 6, 2007, 602. 

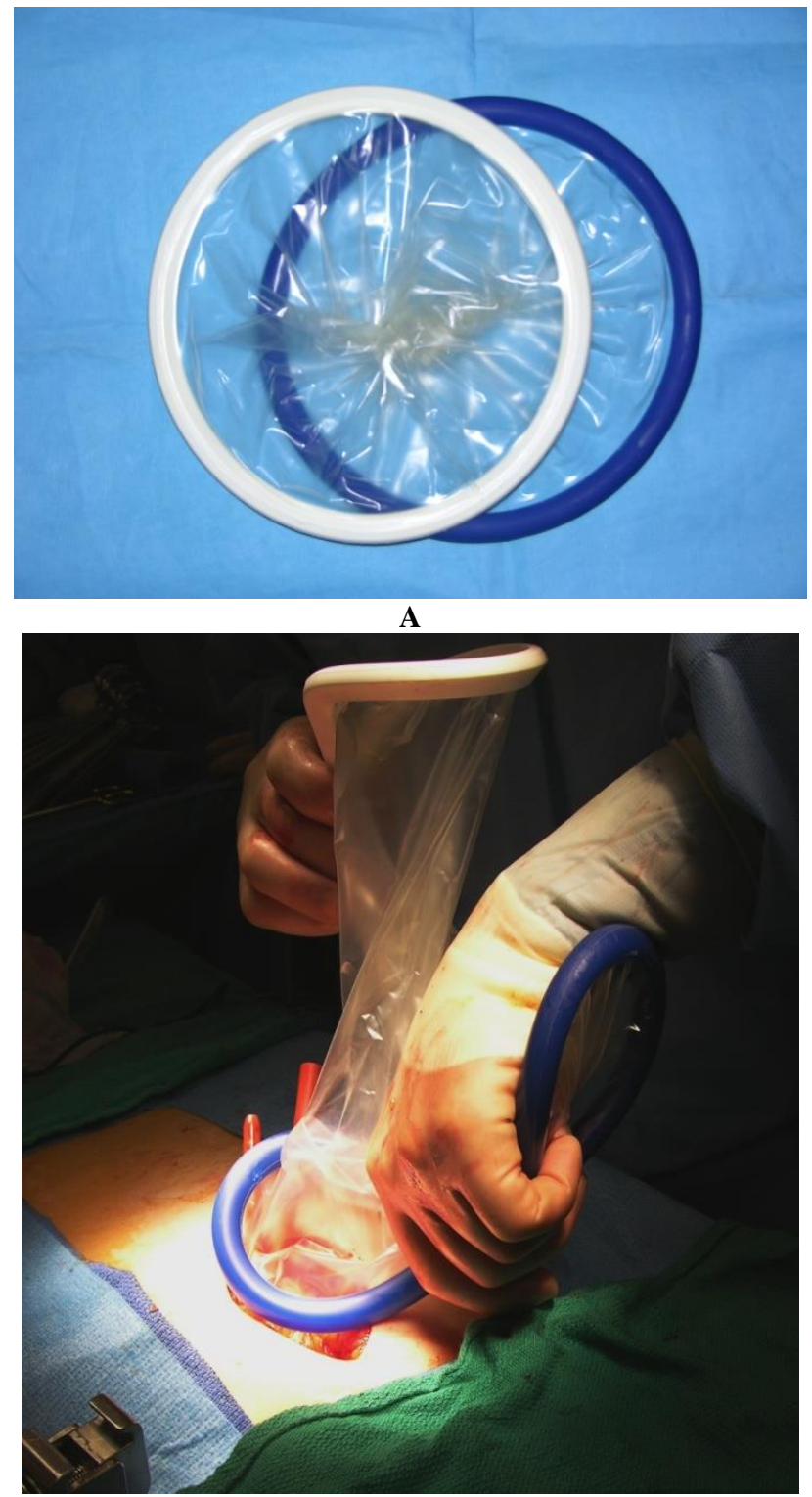

B

Fig. 1. A - "Protractor ring", B - insertion of the ring into the wound 


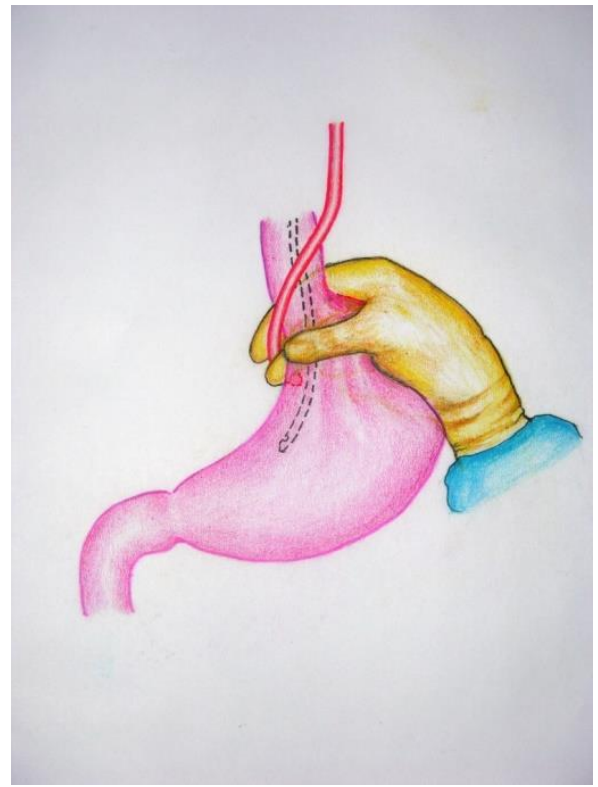

Fig. 2. The formation of a tunnel with the index finger around the esopageal-gastric junction to pass the rubber tube

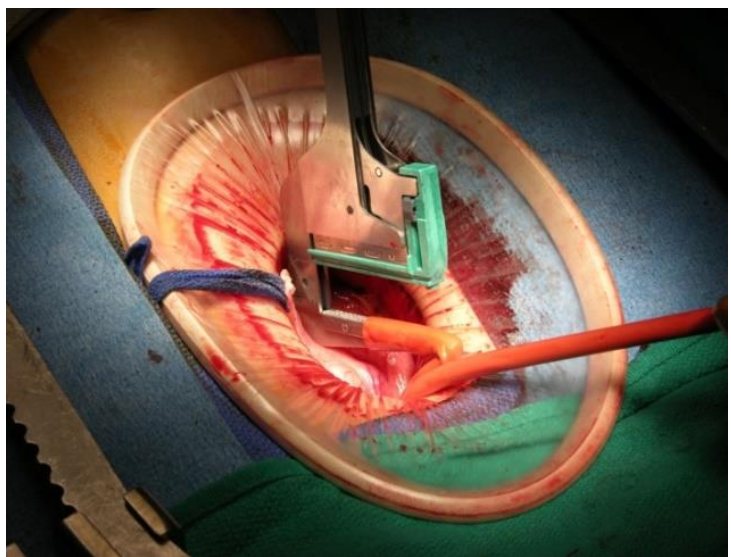

Fig. 3. The stomach is stapled three times

from the gastroesophageal junction towards the angle of His 
When performing the second variant of the formation of a small stomach pouch, stitching and transection of the stomach is carried out using a linear stapler Endo-GIA 45 or Echelon 60 (USA) with flexable cutting jaws. Then a gastrotomy is performed and a circular stapler envil is installed the same way as a first variant

The next stage is bypassing 90-135 $\mathrm{cm}$ of the intestine with the imposition of a stapler jejuno-jejunoanastomosis. Having counted $45 \mathrm{~cm}$ from Treitz's ligament, the jejunum is transected using the Endo-GIA 45 linear stapler?. The mesentery of the small intestine is also dissected $2-3 \mathrm{~cm}$ in the avascular zone (Figure 4).

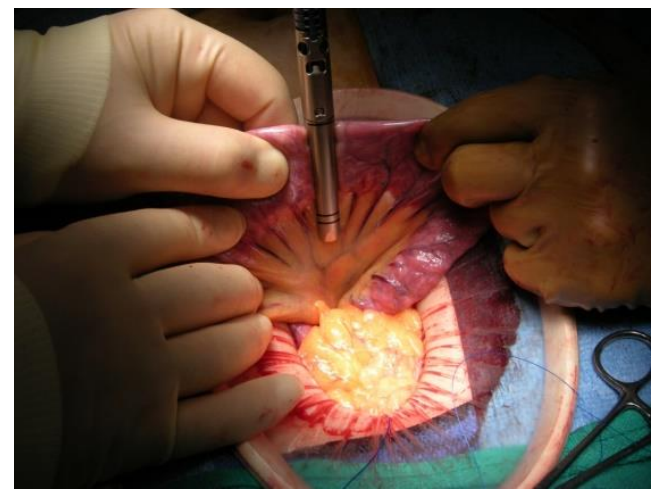

Fig. 4. The jejunum is transected using the Endo-GIA 45 linear stapler

A section of the intestine $(90-135 \mathrm{~cm}$.) for bypassing is measured with $90 \mathrm{~cm}$ tape and a side-to-side stapler jejuno-jejunoanastomosis is formed using Endo-GIA45 stapling in two directions (Figure 5).

The intestinal wound is closed using the TX60 linear stapler. For the prevention of anastomotic leak, separate serous-muscular sutures are applied (Figure 6).

Then the mesenteric defect is closed to prevent internal hernias and intestinal obstruction. The proximal end of the small intestine is then passed through the mesentery behind (retrocolic) or in front (antecolic) of the colon.

${ }^{9}$ Halmi, D, Anez O, Kolesnikov E. 1000 roux-en-y gastric bypass operations through the minilaparotomy incision: efficient, technically simple and cost-effective alternative to the laparoscopic bariatric surgery. XXXV World Congress of the International College of Surgeons, Pattaya, Thailand, October 25-29, 2006, Surgical Challenges, p. 149. 
In most of our cases we applied anterior gastroenteroanastomosis (antegastric) $^{10}$.

The next stage is the opening of the proximal end of the jejunum, the introduction of a circular stapler ILS-21 (or 25) into it and the imposition of gastrojejunostomy side to side. (Figure 7). Performing a gastrojejunal anastomosis using a circular stapler (Figure 8).

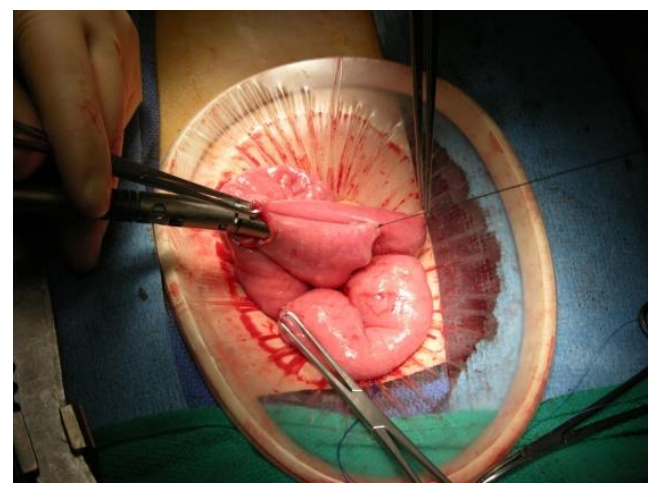

Fig. 5. Jejuno-jejunoanastomosis is formed using Endo-GIA45 stapling in two directions

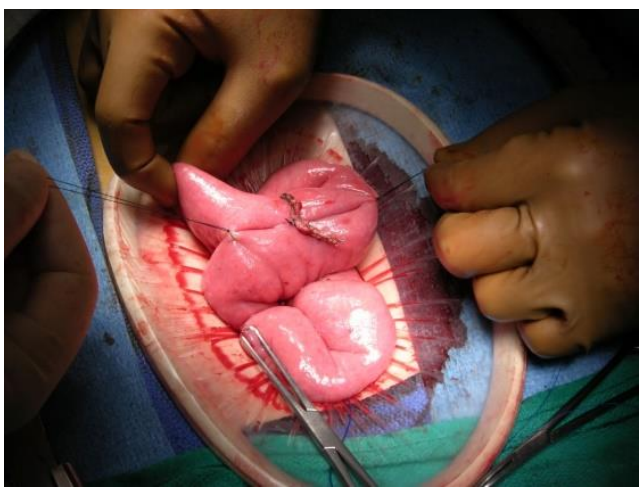

Fig. 6. The intestinal wound is closed using the TX60 linear stapler

${ }^{10}$ Anez O, Halmi D, Kolesnikov E et al. Roux-en-y gastric bypass operation via upper midline minilaparotomy incision: safe, efficient and cost-effective bariatric surgical procedure , XII World Congress of International Federation for the Surgery of Obesity, September 5-8, 2007, Porto, Portugal, Abstracts boor, P. 27. 


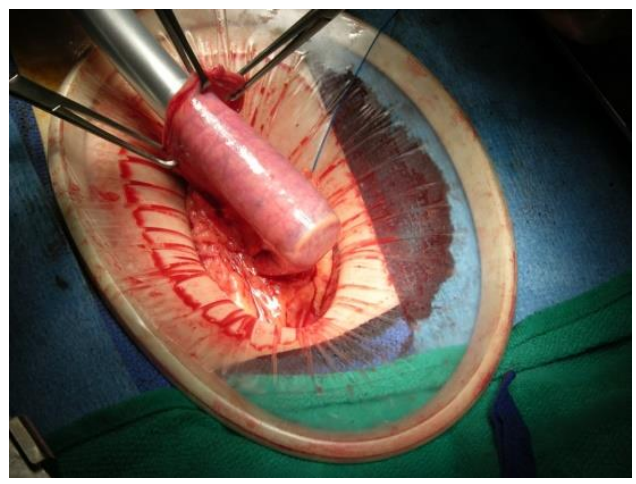

Fig. 7. Introduction of a circular stapler ILS-21 (or 25) into the open jejunum

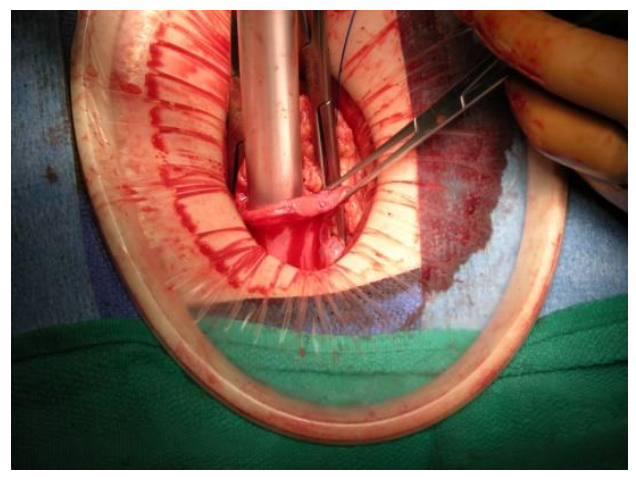

Fig. 8. Performing a gastrojejunal anastomosis using a circular stapler

After removing the circular stapler from the wound, it is necessary to check for the presence of two sections of tissue, cut with the stapler: gastric and intestinal, as shown on the picture (Figure 9).

Then the small intestine is closed with the Endo GIA-45 Flex flexable linear stapler, and reinforcing serous-muscular sutures (3-0 Silk) are applied. (Figure 10).

The control of the consistency of the anastomosis and layer-by-layer suturing of the postoperative wound of the anterior abdominal wall with a cosmetic suture of the skin are performed (Figure 11). 


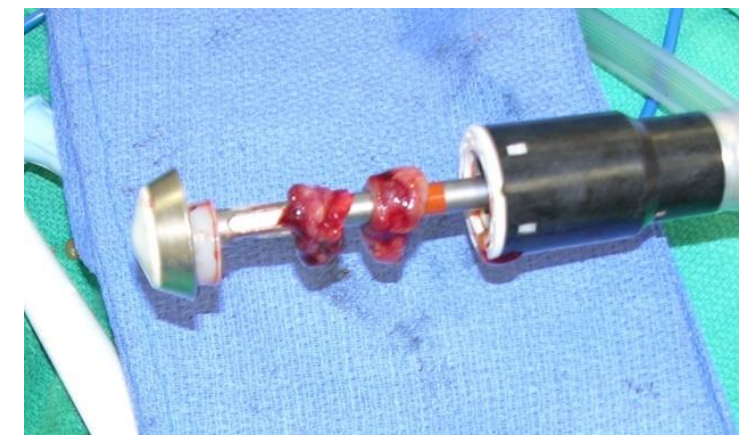

Fig. 9. Checking for the presence of two sections of tissue, cut with the stapler: gastric and intestinal

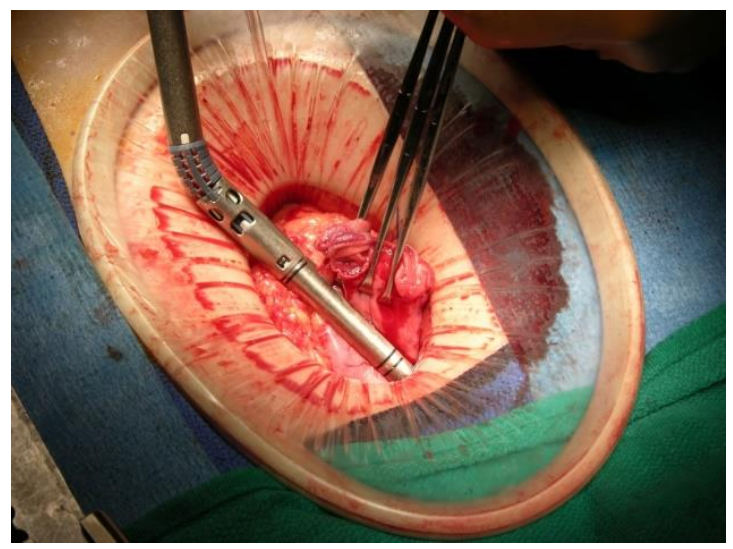

Fig. 10. Open intestine is closed with the Endo GIA-45 Flex linear stapler

When using the laparoscopic technique, all the same stages of the operation were performed using elongated, specially designed for bariatric surgery, laparoscopic instruments: hepatic retractor, trocars - VISIPORT 5-11 mm, Auto Suture (USA); - ENDOPATH XCel $12 \mathrm{~mm}$ and $5 \mathrm{~mm}$ (ETHICON ENDO-SURGERY), - Blunt tip OMS-T10BT $10 \mathrm{~mm}$ (Auto Suture, USA); linear staplers - ECHELON 60, ENDOPATH (ETHICON ENDO-SURGERY), - Endo-GIA45, suture tools - ENDO STITCH $10 \mathrm{~mm}$ (Auto Suture, USA) with 2-0 SOFSILK suture, circular staplers - ILS 25 DST EEA XL (Auto Suture, USA) and other instruments. On the scheme shown the minimally invasive gastrojejunal bypass surgery with Roux-en-Y anastomosis (Figure 12). 


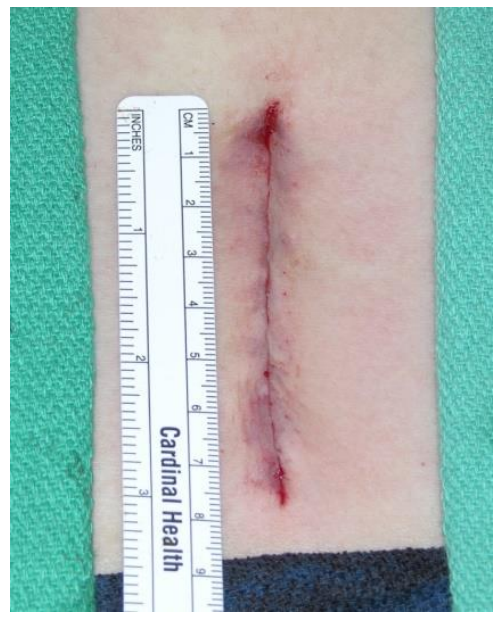

Fig. 11. Postoperative wound of the anterior abdominal wall is closed with a cosmetic suturing technique

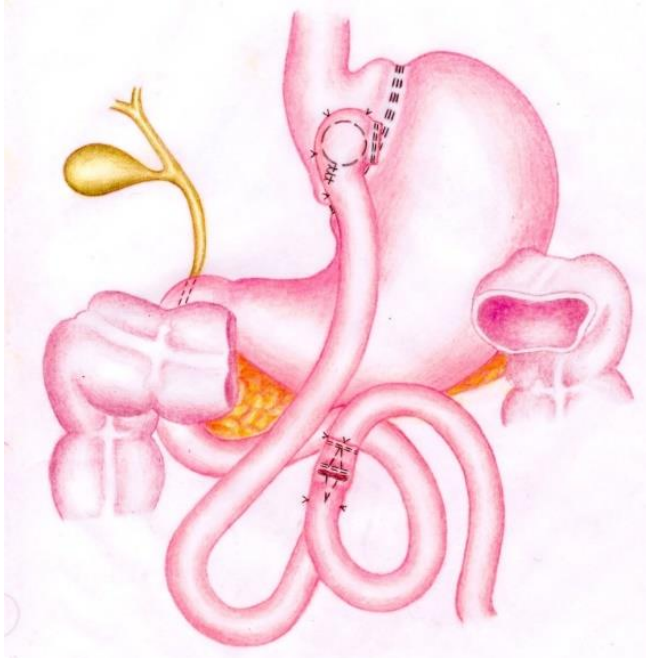

Fig. 12. Scheme of the minimally invasive gastrojejunal bypass surgery with Roux-en-Y anastomosis 


\section{Results and its discussion}

Long-term postoperative complications requiring surgical intervention were observed in $2.9 \%$ of operated patients, of these: - small intestinal obstruction $(0.4 \%)$; - gastro-jejunostomy stricture $(0.2 \%)$; - gastro-gastric fistula $(0.2 \%)$; - incisional hernia $(2.0 \%)$; - dilation of gastro-jejunostomy $(0.1 \%)$; - leakage of the anastomosis $(0.1 \%)$.

The advantages of our proposed surgical technique are: - less surgical trauma due to a small incision $(8 \mathrm{~cm})$ and a reduction in unnecessary manipulations with the intestine; - quickly applied reliable stapler anastomoses, - short operation time (about 1 hour). There were also a low percentage of postoperative complications (early $-1.4 \%$, long-term $-2.9 \%$ ) and mortality $-0.2 \%$. Short hospitalization time -3 days. Relatively short time for mastering the surgical technique (25-30 operations). The number of incisional hernias was $2 \%$.

The costs for performing this operation are much less than using the laparoscopic technique while achieving the same minimal trauma of the operation.

Studies of long-term results after performing this operation showed a decrease in excess weight after one year by $74 \%$, after three years the reduced weight remains by $71 \%$ and after five years the weight remains reduced by $68-70 \%$.

In 2007 our group reported the results of 4155 mini-open surgeries for morbid obesity at the American Congress of US Bariatric Surgeons in San Diego, California and received recognition from our colleagues at this international forum. The results obtained have shown the effectiveness, reproducibility and safety of this operation technique, considered as the "gold standard" in bariatric surgery, obtained both by surgeons in our group and by other surgeons from other bariatric centers who have mastered this method and undergone training in our center ${ }^{11}$.

\section{CONCLUSIONS}

The Roux-en-Y gastrojejunal bypass trough the mini-laparotomy access is safe, simple to perform and cost effective operation. The risk of life threatening complications (leak, obstruction) is lower than with laparoscopic or open approach, but the length of hospitalization is similar with laparoscopic access. With proper training and experience minimally invasive Roux-en-Y gastrojejunal bypass through the mini-laparotomy incision is the

${ }^{11}$ Anez O, Halmi D, Kolesnikov E 4155 minilaparotomy roux-en-y gastric bypass operations: time and volume tested approach for safety, efficacy and efficiency in the treatment of morbid obesity $24^{\text {th }}$ Annual Meeting of the American Society for Bariatric Surgery, San Diego, CA, June 11-16, 2007, USA, Abstract book, p. 67. 
optimal bariatric operation considered as "golden standard" in surgical treatment of morbidly obese patients.

\section{SUMMARY}

Introduction. The most effective treatment of morbidly obese patients is bariatric surgery. The optimal surgical technique of the mini-invasive bariatric interventions is described.

Materials and methods. The paper presents the mini-invasive stapler technique of gastrojejunal bypass surgery through the mini-laparotomy incision. To date, 6,000 patients have undergone mini-invasive bariatric surgery.

Results. The advantages of this method were: - less operational trauma due to a small incision, - quickly applied reliable stapler anastomoses, short operation time. A low percentage of postoperative complications was noted (early $-1.4 \%$, late $-2.9 \%$ ) and mortality $-0.2 \%$. Short hospitalization stay time was 3 days.

Discussion. Studies of long-term results after performing this operation showed a decrease of excess weight in one year by $74 \%$, in three years the reduced weight remains at $71 \%$ and after five years - the weight remains reduced by $68-70 \%$.

Conclusion. The obtained results showed the efficacy, reproducibility, safety and cost-effectiveness considering of this surgical technique as a "golden standard" in bariatric surgery.

Keywords. bariatric surgery, mini-invasive surgeries, gastrojejunal bypass, mini-laparotomy incision, stapler technique.

\section{REFERENCES}

1. Halmi D, Anez O, Kolesnikov E. Mini-open Roux-en-Y gastric bypass is a safe and cost-effective alternative to the open and laparoscopic bariatric surgery. XXXIV World Congress of the International College of Surgeons, October 2004, Quito, Ecuador. International Proceedings MEDIMOND, Italy, 2004, p. 105-108

2. Halmi D, Anez O, Kolesnikov E. Mini-open Roux-en-Y gastric bypass: Complications and Difficulties. XXXIV World Congress of the International College of Surgeons, October 2004, Quito, Ecuador. International Proceedings, MEDIMOND, Italy,2004, p. 109-113

3. Halmi D, Anez O, Kolesnikov E. Mini-open Roux-en-Y Gastric Bypass with simultaneous cholecystectomy for the treatment of morbid obesity and cholelithiasis. Obesity Surgery, 14, 4, 2004, p. 469-70.

4. Halmi D, Anez O, Kolesnikov E. Mini-open Roux-en-Y gastric bypass: 1200 anastomoses without leak. Obesity Surgery, 14, 8, 2004, p. 900-901. 
5. Halmi D, Anez O, Kolesnikov E. Mini-open Roux-en-Y gastric bypass as the operation of choice for the majority of morbidly obese patients. Obesity surgery, 15, 2005, p. 960-1

6. Halmi D, Anez O, Kolesnikov E. Roux-en-y gastric bypass: gastrojejunostomy technique and complications. $2^{\text {nd }}$ European IFSO Congress of Bariatric and Plastic Surgery, April 27-29, 2006, Lyon, France Obesity Surgery, 16, (4) 2006, 404-405.

7. Halmi D, Kolesnikov E. Roux-en-y gastric bypass with concomitant cholecystectomy through the mini-laparotomy incision, 7-th World Congress of the International Hepato-Pancreato Billiary Association in Edinburgh, Scotland, September 3-7, 2006, HPB journal, Vol. 8; No 2 supl, Sept 2006, p. $101-102$.

8. Halmi D, Kolesnikov E. Pre-operative placement of retrievable inferior vena cava filters in bariatric surgery. Surg for Obes and Rel Dis, Vol. 3, No.6, 2007, 602-605

9. Halmi, D, Anez O, Kolesnikov E. 1000 roux-en-y gastric bypass operations through the minilaparotomy incision: efficient, technically simple and cost-effective alternative to the laparoscopic bariatric surgery. XXXV World Congress of the International College of Surgeons, Pattaya, Thailand, October 25-29, 2006, Surgical Challenges, p. 149-151

10. Anez O, Halmi D, Kolesnikov E et al. Roux-en-y gastric bypass operation via upper midline minilaparotomy incision: safe, efficient and cost-effective bariatric surgical procedure, XII World Congress of International Federation for the Surgery of Obesity, September 5-8, 2007, Porto, Portugal, Abstract book, P. 27-28

11. Anez O, Halmi D, Kolesnikov E 4155 minilaparotomy roux-en-y gastric bypass operations: time and volume tested approach for safety, efficacy and efficiency in the treatment of morbid obesity $24^{\text {th }}$ Annual Meeting of the American Society for Bariatric Surgery, San Diego, CA, June 11-16, 2007, USA, Abstract book, P. 67-69.

Information about the author: Kolesnykov Yevhenii Borysovych, orcid.org/0000-0001-5582-7580

Doctor of Medical Sciences, Professor, Professor at the Department of General and Emergency Surgery Shupyk National Healthcare University of Ukraine 9, Dorohozhytska str., Kyiv, 04112, Ukraine 